\title{
DESCRIPTION OF SOME WEIGHTED EXPONENTIAL CLASSES OF SUBHARMONIC FUNCTIONS
}

\author{
O.V. Okhlupina \\ Bryansk State engineering-technological University, Bryansk, Russian Federation \\ E-mail: helga131081@yandex.ru
}

The role of subharmonic functions in such sections of analysis as complex and real analysis is very significant. Such classes of functions are closely related to analytic harmonic functions and make an important contribution to the general theory of potential and mathematical physics. In the works of R. Nevanlinna and $W$. Heiman, parametric representations of subharmonic classes in the plane of functions, whose characteristic has a power growth at infinity, are obtained. The question of whether similar representations are true for weighted classes that admit a stronger growth at infinity (for example, the exponential growth) arises in the theory of entire and meromorphic functions. In this article, classes of subharmonic functions with Nevanlinna characteristic that is summable with exponential weight in a complex plane are introduced for consideration, and the representing measures of functions of such classes are studied. When proving the results, methods of complex and functional analysis are used. An important role in the study is played by potentials based on the factors of the modified Weierstrass product. The proof of the main result is based on the use of auxiliary assertions formulated in the form of lemmas.

Keywords: subharmonic function; harmonic function; representing measures; the Nevanlinna's characteristic.

\section{Introduction}

Let $C$ be the complex plane. $S H_{\alpha, \sigma}(C)$ ( $\sigma, \alpha$ are positive numbers) be the class of subharmonic functions $u$ in $C$ such that $\int_{1}^{+\infty} T(r, u) e^{-\sigma r^{\alpha}} d r<+\infty$, where $T(r, u)$ is the Nevanlinna's characteristic of subharmonic function $u$, that is $T(r, u)=\frac{1}{2 \pi} \int_{-\pi}^{\pi} u^{+}(r \cos \varphi, r \sin \varphi) d \varphi, u^{+}=\max (u, 0)$ (see [1-4]).

In complex and real analysis, potential theory and mathematical physics the value of subharmonic functions is very significant (see [1-3, 5]). In the works R. Nevanlinna and W. Hayman (e.g., see [1]) the obtained definition of a class of subharmonic in the plane of the functions, the characteristics of which have exponential growth at infinity. The question of whether faithful same parametric representation for the weight classes, allowing for stronger growth at infinity, say exponential growth, occurs in the theory of entire and meromorphic functions (see [6]).

This paper studied the representing measures of the functions of class $S H_{\alpha, \sigma}(C)$, as well as the necessary and sufficient condition for such measures.

\section{Statement of the main result}

Let $\sigma, \alpha>0, z, \zeta \in C, \zeta \neq 0, p(|\zeta|)=\max \left[\sigma|\zeta|^{\alpha}, 1\right]$, where $[a]$ is the integer part of a real number $a$.

$$
A_{p}(z, \zeta)=\left(1-\frac{z}{\zeta}\right) \exp \left(\sum_{j=1}^{p(|\zeta|)} \frac{1}{j}\left(\frac{z}{\zeta}\right)^{j}\right) \text { is a factor of the modified product by K. Weierstrass (see }
$$




\section{Математика}

Theorem 1. Let $u \in S H_{\alpha, \sigma}(C), \mu$ is representing measure of a function $u$. Then $\mu$ satisfies the condition:

$$
\int_{1}^{+\infty} \frac{n(t) e^{-\sigma t^{\alpha}}}{t^{\alpha}} d t<+\infty,
$$

where $n(t)=\mu\left(D_{t}\right),|z|<t$.

The opposite is also true: let $\mu$ is some non-negative Borel measure in $C$, satisfying the condition (1), then it is possible to build explicitly subharmonic function of class $S H_{\alpha, \sigma^{\prime}}(C)$, $\forall \sigma^{\prime}: \sigma^{\prime}>\frac{1}{e \alpha}+\sigma e^{\alpha}$, for which $\mu$ will be a representing measure.

\section{Proof of auxiliary assertions}

We require some auxiliary assertions for the proof of the theorem.

Lemma 1. Let $\psi(R)$ is a non-negative monotonically increasing function, for which

$$
\int_{1}^{+\infty} \psi(x) e^{-\sigma x^{\alpha}} d x<+\infty,
$$

where $\alpha>0$. Then

$$
\lim _{R \rightarrow \infty} \frac{\psi(R) e^{-\sigma R^{\alpha}}}{R^{1-\alpha}}=0
$$

Proof. The convergence of the integral (2) implies that the $\lim _{R \rightarrow+\infty} \int_{R}^{+\infty} \psi(x) e^{-\sigma x^{\alpha}} d x=0$.

Let $\varphi(R)=\int_{R}^{+\infty} \psi(x) e^{-\sigma x^{\alpha}} d x$. It is clear that $\varphi(R) \geq \psi(R) \int_{R}^{+\infty} e^{-\sigma x^{\alpha}} d x$. We find the asymptotics of the last integral by applying the L'Hospital's rule.

$$
\lim _{R \rightarrow \infty} \frac{\int_{R}^{+\infty} e^{-\sigma x^{\alpha}} d x}{\frac{e^{-\sigma R^{\alpha}}}{R^{\alpha-1}}}=\lim _{R \rightarrow \infty} \frac{e^{-\sigma R^{\alpha}}}{\left(\frac{e^{-\sigma R^{\alpha}} \sigma R^{\alpha-1} \alpha}{R^{\alpha-1}}+\frac{e^{-\sigma R^{\alpha}}(\alpha-1)}{R^{\alpha}}\right)}=\frac{1}{\sigma \alpha} .
$$

Therefore, $\lim _{R \rightarrow \infty} \frac{\psi(R) e^{-\sigma R^{\alpha}}}{R^{1-\alpha}}=0$. That is $\lim _{R \rightarrow \infty} \psi(R) e^{-\sigma R^{\alpha}} R^{\alpha-1}=0$.

The lemma is proved.

Lemma 2. Let $\varphi(x)=e^{-\sigma x^{\alpha}}, x \in R_{+}, R_{+}=\{x \in R: x \geq 0\}, \varepsilon_{x}=\frac{1}{x^{\alpha}}$. Then $\varphi\left(x+\varepsilon_{x}\right)=e^{\varphi(x)} \cdot e^{\beta(x)}$, where $\beta(x)=o\left(\frac{1}{x}\right)$ when $x \rightarrow+\infty$.

Proof. It follows easily from the following simple arguments. It is clear that $(1+y)^{\alpha}=1+\alpha y+o\left(y^{2}\right)$ when $y \rightarrow 0$. So $\sigma\left(x+\varepsilon_{x}\right)^{\alpha}=\sigma x^{\alpha}\left(1+\frac{\varepsilon_{x}}{x}\right)^{\alpha}=\sigma x^{\alpha}+\frac{\alpha \sigma}{x}+o\left(\frac{1}{x^{\alpha+2}}\right)$ when $x \rightarrow+\infty$.

Then, $e^{\varphi\left(x+\varepsilon_{x}\right)}=e^{\varphi(x)} \cdot e^{\beta(x)}$, where $\beta(x)=o\left(\frac{1}{x}\right)$ when $x \rightarrow+\infty$.

The lemma is proved. 
Lemma 3. Let $u$ is an arbitrary subharmonic function in $C$, while it admits a representation in the form:

$$
u(z)=\int_{C} \ln \left|A_{p}(z, \zeta)\right| d \mu(\zeta)+h(z)
$$

$z, \zeta \in C, \mu(\zeta)$ is an arbitrary non-negative Borel measure in $C$, such that $\int_{1}^{+\infty} \frac{n(t) e^{-\sigma t^{\alpha}}}{t^{\alpha}} d t<+\infty$, $n(r)=\mu\left(D_{r}\right), \sigma>0, \alpha>0, h(z)$ is harmonic function in $C$, for which

$$
\int_{1}^{+\infty} e^{-\sigma r^{\alpha}} \int_{-\pi}^{\pi}\left|h\left(r e^{i \varphi}\right)\right| d \varphi d r<+\infty
$$

Then $u \in S H_{\alpha, \sigma^{\prime}}(C)$ when $\forall \sigma^{\prime}: \sigma^{\prime}>\frac{1}{e \alpha}+\sigma e^{\alpha}$.

\section{Proof.}

Let $V_{p}(z)=\int_{C} \ln \mid A_{p}(z, \zeta) d \mu(\zeta)$. Then $u(z)=h(z)+V_{p}(z)$.

It is obvious that $h(z)$ belongs to the class under consideration. We will show that $V_{p}(z)$ also included in the class $S H_{\alpha, \sigma^{\prime}}(C)$ for $\forall \sigma^{\prime}=\sigma^{\prime}(\alpha)>\sigma$.

We apply the estimate (see [1], p. 94):

$$
\ln \left|A_{p}(z, \zeta)\right| \leq\left|\frac{z}{\zeta}\right|^{p(|\zeta|)}, \zeta \neq 0, z, \zeta \in C
$$

Let's get that: $u(z) \leq \int_{C}\left|\frac{z}{\zeta}\right|^{p(\mid \zeta))} d \mu(\zeta)$.

To continue the evaluation of the function, we can partition the complex plane into sets: $\Delta_{k}=\left\{z \in C: 2^{k}<|z| \leq 2^{k+1}\right\}, \Delta_{0}=\{z \in C:|z| \leq 1\}$. Then $u(z) \leq \sum_{k=0}^{+\infty} \int_{\Delta_{k}}\left|\frac{z}{\zeta}\right|^{p(|\zeta|)} d \mu(\zeta)$.

Each of the rings $\Delta_{k}$ is divided into small rings and we use Lemma 2.

Let $\delta_{k, j}=\left\{z \in C: 2^{k}+j 2^{-\alpha k}<|z| \leq 2^{k}+(j+1) 2^{-k \alpha}\right\}, 0 \leq j \leq\left[2^{k(\alpha+1)+1}\right]$, where $[a]$ is the integer part of the number $a$. Then $\Delta_{k} \subset \bigcup_{j=0}^{N_{\alpha}} \delta_{k, j}$, where $N_{\alpha}=\left[2^{k(\alpha+1)+1}\right]$.

Therefore, $\int_{\Delta_{k}}\left|\frac{z}{\zeta}\right|^{p(|\zeta|)} d \mu(\zeta) \leq \sum_{j=0}^{N_{\alpha}} \int_{\delta_{k, j}}\left|\frac{z}{\zeta}\right|^{p(|\zeta|)} d \mu(\zeta)$.

By Lemma 2, we obtain

$\int_{\delta_{k, j}}\left|\frac{z}{\zeta}\right|^{p(|\zeta|)} d \mu(\zeta) \leq\left|\frac{z}{\zeta^{*}}\right|^{p\left(\mid \zeta^{*}\right)} d \mu\left(\delta_{k, j}\right) \leq\left|\frac{z}{\zeta^{*}}\right|^{p\left(\mid \zeta^{*}\right)} n\left(2^{k}+(j+1) 2^{-\alpha k}\right)$, where $\zeta^{*}$ is a point of $\delta_{k, j}$. It is obvious that $\left|\frac{z}{\zeta^{*}}\right|^{p\left(\left|\zeta^{*}\right|\right)} n\left(2^{k}+(j+1) 2^{-\alpha k}\right) \leq C_{0} \int_{2^{k}+(j+1) 2^{-\alpha k}}^{2^{k}+(j+2) 2^{-\alpha k}}\left|\frac{z}{t}\right|^{p(|t|)} n(t) d t, k=0,1, \ldots$

Therefore $\int_{\Delta_{k}}\left|\frac{z}{\zeta}\right|^{p(|\zeta|)} d \mu(\zeta) \leq C \sum_{j=0}^{N_{\alpha}} \int_{\delta_{k, j}}^{\delta_{k, j+1}}\left|\frac{z}{t}\right|^{p(t)} n(t) d t \leq C \int_{2^{k}}^{2^{k+1}}\left|\frac{z}{t}\right|^{p(t)} n(t) d t$.

Summing over $k$, we get: 


$$
u(z) \leq C \int_{1}^{+\infty}\left|\frac{z}{t}\right|^{p(t)} n(t) d t .
$$

Let us estimate the last integral. According to (5) we have that

$$
\int_{1}^{+\infty} e^{-\sigma^{\prime} r^{\alpha}} \int_{-\pi}^{\pi} u^{+}\left(r e^{i \varphi}\right) d \varphi d r \leq C \int_{1}^{+\infty} e^{-\sigma^{\prime} r^{\alpha}} \int_{1}^{+\infty}\left(\frac{r}{t}\right)^{p(t)} n(t) d t d r .
$$

Having established the convergence of the last integral, we prove the Lemma.

To do this, imagine the inner integral as a sum:

$$
\int_{1}^{+\infty}\left(\frac{r}{t}\right)^{p(t)} n(t) d t=\int_{1}^{e r}\left(\frac{r}{t}\right)^{p(t)} n(t) d t+\int_{e r}^{+\infty}\left(\frac{r}{t}\right)^{p(t)} n(t) d t=I_{1}+I_{2} .
$$

Let us prove the boundedness of the second integral by a constant, independently of $r$. Indeed, $\left(\frac{r}{t}\right)^{p(t)}=\exp p(t) \ln \frac{r}{t}$.

However, when $\frac{r}{t} \leq e^{-1} \ln \frac{r}{t} \leq-1$. Then $\exp p(t) \ln \frac{r}{t} \leq \exp (-p(t))$.

$p(t)=\left[\sigma^{\prime} t^{\alpha}\right]$, so $p(t) \geq \sigma^{\prime} t^{\alpha}-1$. Therefore, $\exp (-p(t)) \leq C e^{-\sigma^{\prime} t^{\alpha}}$.

$$
\begin{gathered}
e^{-\sigma^{\prime} t^{\alpha}}<\frac{e^{-\sigma t^{\alpha}}}{t^{\alpha}}, \sigma^{\prime}>\sigma, n(t) \leq e^{\sigma t^{\alpha}} t^{2 \alpha}, \\
\int_{e r}^{+\infty}\left(\frac{r}{t}\right)^{p(t)} n(t) d t \leq C \int_{e r}^{+\infty} n(t) e^{-\sigma^{\prime} t^{\alpha}} d t<C \int_{e r}^{+\infty} \frac{n(t) e^{-\sigma t^{\alpha}} d t}{t^{2 \alpha}}<+\infty .
\end{gathered}
$$

We estimate the integral.

$$
I_{1}=\int_{1}^{e r}\left(\frac{r}{t}\right)^{p(t)} n(t) d t
$$

Consider the estimate for the integral $I_{2}$ we get that

$$
\int_{1}^{+\infty} e^{-\sigma^{\prime} r^{\alpha}} \int_{-\pi}^{\pi} u^{+}\left(r e^{i \varphi}\right) d \varphi \leq \int_{1}^{+\infty} e^{-\sigma^{\prime} r^{\alpha}} \int_{1}^{e r}\left(\frac{r}{t}\right)^{p(t)} n(t) d t d r .
$$

To calculate the largest value of the function $\exp p(t)(\ln r-\ln t)$ on an interval [1;er] we put $x=\ln r$ и $\gamma(x, t)=p(t)(x-\ln t)$.

$$
\gamma^{\prime}(x, t)=p^{\prime}(t)(x-\ln t)-\frac{p(t)}{t}=\sigma \alpha t^{\alpha-1}(x-\ln t)-\sigma t^{\alpha-1}=\sigma \alpha t^{\alpha-1}\left(x-\ln \left(t e^{\frac{1}{\alpha}}\right)\right)=0 .
$$

That is $\ln r=\ln \left(t e^{\frac{1}{\alpha}}\right), t=r e^{-\frac{1}{\alpha}}, t^{\alpha}=r^{\alpha} e^{-1}$. This point is the maximum point, therefore $\gamma(x, t)=\gamma\left(x, r e^{-\frac{1}{\alpha}}\right)=p\left(r e^{-\frac{1}{\alpha}}\right)\left(\ln r-\ln r e^{-\frac{1}{\alpha}}\right)$.

Because max $\left(\frac{r}{t}\right)^{p(t)} \leq\left(\frac{r}{r e^{-\frac{1}{\alpha}}}\right)^{\frac{r^{\alpha}}{e}}$, then 


$$
\begin{aligned}
& \int_{1}^{e r} n(t)\left(\frac{r}{t}\right)^{p(t)} d t \leq \int_{1}^{e r} n(t)\left(e^{\frac{1}{\alpha}}\right)^{\frac{r^{\alpha}}{e}} d t=\int_{1}^{e r} n(t) e^{\frac{r^{\alpha}}{\alpha e}} d t=e^{\frac{r^{\alpha}}{\alpha e}} \int_{1}^{e r} n(t) d t \\
& \int_{1}^{+\infty} e^{-\sigma^{\prime} r^{\alpha}} e^{\frac{r^{\alpha}}{e \alpha}} \int_{1}^{e r} n(t) d t d r=\int_{1}^{+\infty} e^{-\left(\sigma^{\prime}-\frac{1}{e \alpha}\right) r^{\alpha}} \int_{1}^{e r} n(t) d t d r= \\
& =\left.C_{1} \int_{1}^{e r} n(t) d t \cdot e^{-\left(\sigma^{\prime}-\frac{1}{e \alpha}\right) r^{\alpha}}\right|_{1} ^{+\infty}+\frac{1}{\alpha} \int_{1}^{+\infty} \frac{e^{-\left(\sigma^{\prime}-\frac{1}{e \alpha}\right) r^{\alpha}}}{\left(\sigma^{\prime}-\frac{1}{e \alpha}\right) r^{\alpha-1}} n(e r) d r .
\end{aligned}
$$

In view of Lemma 1, we write that $\int_{1}^{e r} n(t) d t \leq C e^{\sigma(e r)^{\alpha}} r^{\alpha-1}$.

Using the last estimate, we clarify the values $\sigma^{\prime}$, that ensure the convergence of the integral:

$$
\int_{1}^{+\infty} e^{-\left(\sigma^{\prime}-\frac{1}{e \alpha}\right) r^{\alpha}} \int_{1}^{e r} n(t) d t d r: \sigma^{\prime} r^{\alpha}-\sigma(e r)^{\alpha}-\frac{r^{\alpha}}{\alpha e}>0, r^{\alpha}\left(\sigma^{\prime}-\sigma e^{\alpha}-\frac{1}{\alpha e}\right)>0, \sigma^{\prime}>\frac{1}{e \alpha}+\sigma e^{\alpha} \text {. }
$$

The lemma is proved.

Let us prove the auxiliary theorem 2 .

Theorem 2. Let $u$ is an arbitrary subharmonic function in the class $S_{\sigma, \alpha}(C), \mu(\zeta)$ is an arbitrary measure of the function $u$, in this case $\mu\left(D_{t}\right)=n(t), 0<t<+\infty$. Then

$$
\int_{1}^{+\infty} \frac{n(t) e^{-\sigma t^{\alpha}}}{t^{\alpha}} d t<+\infty .
$$

Proof. We integrate the integral by parts. Using the equality of Jensen for subharmonic functions (see [1]), it is enough to estimate the integral:

$$
I=\int_{1}^{+\infty}\left(\int_{1}^{t} \frac{n(r) d r}{r}\right) e^{-\sigma t^{\alpha}} d t
$$

We integrate $I$ by parts:

$$
I=-\left.\frac{1}{\alpha \sigma}\left(\frac{e^{-\sigma t^{\alpha}}}{t^{\alpha-1}} \int_{1}^{t} \frac{n(r) d r}{r}\right)\right|_{1} ^{+\infty}+\int_{1}^{+\infty} e^{-\sigma t^{\alpha}}\left(\frac{1-\alpha}{t^{\alpha}}\left(\int_{1}^{t} \frac{n(r) d r}{r}\right)+\frac{n(t)}{t^{\alpha}}\right) d t
$$

According to Lemma 1, we have:

$$
I=\int_{1}^{+\infty} e^{-\sigma t^{\alpha}}\left(\frac{1-\alpha}{t^{\alpha}} \int_{1}^{t} \frac{n(r) d r}{r}+\frac{n(t)}{t^{\alpha}}\right) d t=\frac{1}{\alpha \sigma}(1-\alpha) \int_{1}^{+\infty} \frac{e^{-\sigma t^{\alpha}}}{t^{\alpha-1}}\left(\int_{1}^{t} \frac{n(r) d r}{r}+n(t)\right) d t
$$

By the hypothesis of the theorem $I \leq C$.

It is clear that in $0 \leq \alpha<1$ the theorem is proved, since from the condition (5) implies that $\int_{1}^{+\infty} \frac{e^{-\sigma t^{\alpha}}}{t^{\alpha}} n(t) d t<+\infty$.

Suppose $\alpha>1$. Then by Lemma 1:

$$
\int_{1}^{+\infty} \frac{e^{-\sigma t^{\alpha}}}{t^{\alpha}} \int_{1}^{t} \frac{n(r)}{r} d r \leq C_{1} \int_{1}^{+\infty} \frac{e^{-\sigma t^{\alpha}}}{t^{\alpha}} \frac{d t}{e^{-\sigma t^{\alpha}} t^{\alpha-1}}=C_{1} \int_{1}^{+\infty} \frac{d t}{t^{2 \alpha-1}}<+\infty .
$$

Because $\alpha>1$, then $\int_{1}^{+\infty} \frac{e^{-\sigma t^{\alpha}}}{t^{\alpha}} n(t) d t<+\infty$.

The theorem is proved. 


\section{Математика}

\section{The proof of the main result}

The proof of the direct assertion of theorem 1 follows from theorem 2.

The validity of the converse follows from Lemma 3.

That proves theorem 1 completely.

From Theorem 1 directly follows

Theorem 3. Let $\alpha>0, S H_{\alpha, \infty}=\bigcup_{\sigma>0} S H_{\alpha, \sigma}$. Then class $S H_{\alpha, \infty}$ coincides with the class of subharmonic functions in $C$, representable in the form $u(z)=\int_{C} \ln \left|A_{p}(z, \zeta)\right| d \mu(\zeta)+h(z)$, where $\mu$ is nonnegative measure in $C$, such that $n(t)=\mu\left(D_{t}\right), D_{t}=\{z:|z|<t\}$, and also satisfies the condition $\int_{1}^{+\infty} \frac{n(t) e^{-\sigma t^{\alpha}}}{t^{\alpha}} d t<+\infty$ for some $\alpha \geq 0, h$ an arbitrary harmonic function in $C$, for which there exists $\sigma>0: \int_{1}^{+\infty} e^{-\sigma r^{\alpha}} \int_{-\pi}^{\pi}\left|h\left(r e^{i \varphi}\right)\right| d \varphi d r<+\infty$

\section{References}

1. Hayman W.K. Subharmonic functions. Acad. Press, London etc., 1989, Vol. 2, 591 p.

2. Ronkin L.I. Vvedenie v teoriiu tselykh funktsii mnogikh peremennykh (Introduction to the theory of entire functions of several variables). Moscow, Nauka Publ., 1971, 432 p. (in Russ.).

3. Azarin V.S. Teoriia rosta subgarmonicheskikh funktsii (The theory of growth of subharmonic functions). Kharkov, KhGU Publ., 1982, 73 p. (in Russ.).

4. Ohlupina O.V. Potentsialy tipa Grina i integralnye predstavleniia vesovykh klassov subgarmonicheskikh funktsii: dissertatsiia kandidata fiziko-matematicheskikh nauk (Potentials of Green's type and integral representations of the weight classes of subharmonic functions: dissertation of the candidate of physical and mathematical sciences), Briansk, 2012, 118 p. (in Russ.).

5. Brelo M. Osnovy klassicheskoi teorii potentsiala (Fundamentals of classical potential theory), Moscow, Mir Publ., 1964, 215 p. (in Russ.). [Brelot M. Elements de la théorie classique du potentiel, Paris, 1961, 191 p. (in Fr.)]

6. Bykov S.V., Shamoian F.A. O nuliakh tselykh funktsii s mazhorantoi beskonechnogo poriadka (On the zeros of entire functions with majorant of infinite order). Algebra i analiz. Spb, Nauka Publ., 2009, Vol. 21, no. 6, pp. 66-79. (in Russ.).

7. Shamoyan F.A., Djrbashian A.E. Topics in the theory of $A_{\alpha}^{p}$ spaces. Teubner Texte zur Mathematik [Teubner Texts in Mathematics], BSB B.G. Teubner Verlagsgesellschaft, Leipzig, 1988, Vol. 105, p. 200.

8. Shamoian F.A., Shubabko E.N. Vvedenie v teoriiu vesovykh $L^{p}$-klassov meromorfnykh funktsii [Introduction to the theory of the weight $L^{p}$-classes of meromorphic functions]. Brjansk, RIO BGU Publ., 2009, 153 p. (in Russ.).

Received November 3, 2017 


\section{ОПИСАНИЕ НЕКОТОРЫХ ВЕСОВЫХ ЭКСПОНЕНЦИАЛЬНЫХ КЛАССОВ СУБГАРМОНИЧЕСКИХ ФУНКЦИЙ}

\section{О.В. Охлупина}

Брянский государственный инженерно-технологический университет, г. Брянск, Российская Федерация

E-mail: helga131081@yandex.ru

Роль субгармонических функций в таких разделах анализа, как комплексный и вещественный анализ, весьма существенна. Такие классы функций тесно связаны с аналитическими гармоническими функциями и вносят важный вклад в общую теорию потенциала и математическую физику. В трудах Р. Неванлинны, У. Хеймана получены параметрические представления классов субгармонических в плоскости функций, характеристика которых имеет степенной рост в бесконечности. Вопрос о том, верны ли аналогичные представления для весовых классов, которые допускают более сильный рост в бесконечности (например, экспоненциальный рост), возникает в теории целых и мероморфных функций. В статье введены в рассмотрение классы субгармонических функций с характеристикой Неванлинны, которая суммируема с экспоненциальным весом на комплексной плоскости, а также изучены представляющие меры функций таких классов. При доказательстве результатов применяются методы комплексного и функционального анализа. Существенную роль в исследовании играют потенциалы, построенные на основе факторов модифицированного произведения Вейерштрасса. Доказательство основного результата базируется на использовании вспомогательных утверждений, сформулированных в виде лемм.

Ключевые слова: субгармоническая функиия; гармоническая функция; представляюшие меры; характеристика Неванлинны.

\section{Литература}

1. Hayman, W.K. Subharmonic functions / W.K. Hayman. - Acad. Press, London etc. - 1989. Vol. 2. $-591 \mathrm{p}$.

2. Ронкин, Л.И. Введение в теорию целых функций многих переменных / Л.И. Ронкин. - М.: Наука, 1971. - 432 с.

3. Азарин, В.С. Теория роста субгармонических функций / В.С. Азарин. - Харьков: ХГУ, 1982. $-73 \mathrm{c}$.

4. Охлупина, О.В. Потенциалы типа Грина и интегральные представления весовых классов субгармонических функций: дис. ... канд. физ.-мат. наук / О.В. Охлупина. - Брянск, 2012. - 118 с.

5. Брело, М. Основы классической теории потенциала / М. Брело. - М.: Мир, 1964. - 215 с.

6. Быков, С.В. О нулях целых функций с мажорантой бесконечного порядка / С.В. Быков, Ф.А. Шамоян // Алгебра и анализ. - Санкт-Петербургское отделение Института математики РАН им. В.А. Стеклова, СПб: Наука, 2009. - Т. 21, № 6. - С. 66-79.

7. Shamoyan, F.A. Topics in the theory of $A_{\alpha}^{p}$ spaces / F.A. Shamoyan, A.E. Djrbashian // Teubner Texte zur Mathematik [Teubner Texts in Mathematics]. - BSB B. G. Teubner Verlagsgesellschaft, Leipzig, 1988. - Vol. 105. - P. 200.

8. Шамоян, Ф.А. Введение в теорию весовых $L^{p}$-классов мероморфных функций / Ф.А. Шамоян, Е.Н. Шубабко. - Брянск: РИО БГУ, 2009. - 153 с.

Поступила в редакцию 3 ноября 20172. 